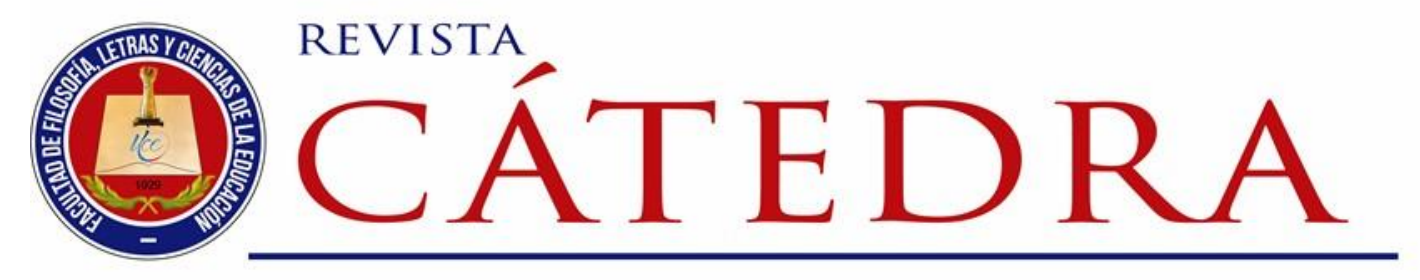

\title{
Perfiles de virtuosidad en organizaciones escolares en relación con los niveles de bienestar psicológico en adolescentes ecuatorianos
}

\section{Virtuousness profiles in school organizations in relations to psychological well-being levels in Ecuadorian adolescents}

\begin{abstract}
Fernando Unda-Villafuerte
Ministerio de Salud Pública del Ecuador, Universidad Central del Ecuador, Quito, Ecuador fsunda@uce.edu.ec https://orcid.org/0000-0002-4958-2529

María Laura Lupano-Perugini Consejo Nacional de Investigaciones Científicas y Técnicas, Universidad de Palermo, Buenos Aires, Argentina mlupan1@palermo.edu https://orcid.org/0000-0001-6090-0762
\end{abstract}

(Recibido: 09/02/2019; Aceptado: 29/03/2019; Versión final recibida: 04/04/2019)

Cita del artículo: Unda-Villafuerte, F. y Lupano-Perugini, M. (2019). Perfiles de virtuosidad en organizaciones escolares en relación con los niveles de bienestar psicológico en adolescentes ecuatorianos. Revista Cátedra, 2(2), 76-93.

\section{Resumen}

El estudio tuvo como objetivos analizar la relación entre virtudes percibidas en organizaciones escolares positivas y los niveles de bienestar psicológico en adolescentes y; establecer perfiles de organizaciones escolares positivas o virtuosas considerando, por un lado, el género y tipo de financiamiento y, por otro, los niveles de bienestar psicológico presentes en adolescentes ecuatorianos. La investigación se justifica por la necesidad de

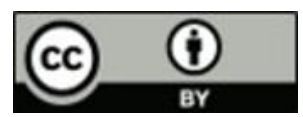


generar espacios y estrategias de socialización y aprendizaje que potencien el desarrollo integral de chicos y chicas y su bienestar perdurable en contextos influenciados por el placer instantáneo y de alta intensidad. Para el estudio se consideró una muestra de 550 estudiantes (51,7\% hombres). Los instrumentos aplicados fueron la escala de virtuosidad percibida en organizaciones escolares y la escala de bienestar psicológico en adolescentes, los mismos se hallan validados a los contextos ecuatorianos. Como resultado del análisis estadístico se evidenció una correlación moderada entre las variables de estudio. Adicionalmente se llevaron a cabos dos procesos estadísticos: análisis de conglomerados y análisis de varianza (ANOVA), de esta forma se establecieron perfiles de bienestar psicológico en adolescentes en función del nivel de virtuosidad percibida en las organizaciones escolares. El análisis mencionado dio lugar a tres niveles de organizaciones escolares (virtuosas, en tránsito a la virtuosidad y poco virtuosos) que interactuaron en su orden con tres niveles de bienestar psicológico (fortalecido, en proceso de construcción y vulnerable). Finalmente, en el acápite conclusiones se formularon estrategias puntuales para generar organizaciones virtuosas vinculadas con mejores niveles de bienestar en estudiantes.

Palabras clave:

Adolescencia, bienestar psicológico, organizaciones escolares, psicología positiva, virtuosidad.

\section{Abstract}

The purpose of this study was to analyze the relationship between perceived virtues in positive school organizations and levels of well-being in adolecents; and, establish profiles of positive or virtuous school organizations considering, on the one hand, the gender and type of funding and, on the other, the levels of psychological well-being present in Ecuadorian adolescents. The research is justified by the need to generate spaces and strategies for socialization and learning that enhance the integral development of boys and girls and their lasting well-being in contexts influenced by instantaneous pleasure and high intensity. For the study, a sample of 550 students $(51.7 \%$ men) was considered. The research applied two instruments validated to Ecuadorian contexts: the scale of perceived virtuousness in school organizations and the scale of psychological well-being in adolescents. As a result of the statistical analysis, a moderate correlation was observed between the study variables. Next, and in order to enhance the analysis, a cluster analysis of average $\mathrm{k}$ and an analysis of variance (ANOVA) of one factor were carried out to establish profiles of psychological well-being in adolescents according to the level of virtuosity perceived in school organizations. The aforementioned analysis gave rise to three levels of school organizations (virtuous, in transit to virtuousness and little virtuousness) that interacted in their order with three levels of psychological well-being (strengthened, under construction and vulnerable). Finally, in the section conclusions, specific strategies were formulated to generate virtuous organizations linked with better levels of well-being in students.

Keywords:

Adolescence, Positive Psychology, psychological well-being, school organizations, virtuousness. 


\section{Introducción}

La literatura científica coincide en que la adolescencia es el período comprendido entre la infancia y la edad adulta caracterizado por un conjunto acelerado de cambios fisiológicos, psicológicos y físicos que inician con la primera eyaculación en los varones y la primera menstruación en las mujeres. El Fondo de las Naciones Unidas para la Infancia (2011) explica que adolescencia es un término difícil de explicar, esto debido a que "la madurez física, emocional y cognitiva, entre otros factores, depende la manera en que cada individuo experimenta este período de la vida" (p.8). En términos operativos y de política pública se reconoce a la adolescencia como el período de vida comprendido entre los 10 y 19 años, definiéndose dos etapas: adolescencia temprana (10 a 14 años) y adolescencia tardía (15 a 19 años). Así mismo, es importante diferenciar entre pubertad y adolescencia, la primera se refiere a "un proceso biológico en el que se produce el desarrollo de los caracteres sexuales secundarios, la maduración de las gónadas y glándulas suprarrenales y la adquisición del pico de masa ósea, grasa y muscular" (Güemes-Hidalgo et al., p. 8); mientras que la segunda tiene que ver con marcadores físicos, psicosociales, emocionales y cognitivos que inician con la pubertad y culminan en la segunda década de la vida. En términos amplios el sistema educativo ecuatoriano contempla el nivel de educación básica superior que se relaciona con la adolescencia temprana y el bachillerato que coincide con la adolescencia tardía.

Los cambios biológicos y psicológicos generados en la adolescencia, bajo la influencia de contextos sociales y culturales específicos, pueden dar lugar a conductas que permiten una vida saludable y un desarrollo integral o, por el contrario, traer como consecuencia conductas de riesgo y poco saludables. Identificar factores protectores o de riesgo constituye un elemento clave en el bienestar integral de la adolescencia; sin embargo, González y Rivera (2016) acotan que "el ajuste o desajuste psicológico, no está predeterminado por la presencia de riesgos en el desarrollo, sino por la combinación de variables que en su conjunto pueden culminar en patrones adaptativos como desadaptativos" (p. 48).

En las sociedades contemporáneas la familia, la escuela, el grupo de amigos y la interacción virtual constituyen espacios esenciales de socialización. En la adolescencia se evidencia un paulatino cuestionamiento a la familia y sus normas y un acercamiento a los grupos de pares mediante mecanismos virtuales y de contacto directo. Muchos de los procesos de socialización tienen lugar en la escuela, misma que "impone a sus alumnos un modo de ser y de actuar, una moralidad y unos valores, su función principal será la de educar no sólo en la versión académica del concepto sino también moral, social y normativa" (Navarro-Pérez et al., 2015, p. 147). La perspectiva ecológica planteada determina que la escuela, la familia y las políticas educativas concreten estrategias que deriven en el desarrollo integral de los adolescentes.

La escuela positiva, como espacio de desarrollo integral de los adolescentes, busca fortalecer destrezas, habilidades y competencias que redunden en el bienestar psicológico de chicos y chicas entendido como prácticas, actitudes y conocimientos que posibiliten mejoras niveles de satisfacción personal y social. Sin embargo, "entornos educativos contaminados generan en el adolescente angustia, frustración, dificultan el aprendizaje y su socialización" (Navarro-Pérez et al., p. 147). Tendencias actuales de la psicología educativa, la psicopedagogía y la psicología evolutiva plantean que el bienestar psicológico y la satisfacción por la vida en la adolescencia pasa por "conocer los aspectos positivos y fortalecer aquellos factores que pueden ayudar a mejorar sus mecanismos de adaptación y 
su salud integral, en términos de bienestar físico y emocional, que permitan prosperar como individuos plenos dentro de sus comunidades" (Barcelata y Rivas, 2016, p. 120).

En la presente investigación los establecimientos educativos que posibilitan el bienestar psicológico, como parte del desarrollo integral de los adolescentes, se definen como organizaciones escolares positivas o virtuosas; mientras que aquellos establecimientos que no vinculan en sus prioridades el bienestar psicológico de sus estudiantes se consideran organizaciones escolares no virtuosas. En cualquier caso, se parte de la comprensión de la escuela como un espacio privilegiado de socialización de los adolescentes que influirá decididamente en su crecimiento y desarrollo.

El marco de análisis de las variables (virtuosidad percibida en organizaciones escolares y bienestar psicológico en adolescentes) consideró los principios teóricos y fundamentos de la Psicología Positiva entendida como el abordaje científico de la experiencia humana desde "la vida placentera que da lugar al estudio de las emociones positivas; la vida comprometida relacionada con el estudio de las fortalezas y las virtudes personales; $y$, el funcionamiento de las instituciones positivas" (Lupano-Perugini y Castro-Solano, 2010, p. 48). La opción por este enfoque se basó en la necesidad de contar con un marco referencial válido y actualizado que permita identificar alternativas científicas destinadas a potenciar el desarrollo integral de los adolescentes e incidir de manera positiva sobre sus espacios cotidianos de socialización.

De esta forma, el estudio realizado tuvo como objetivos analizar la relación entre virtudes percibidas en organizaciones escolares positivas y los niveles de bienestar y; establecer perfiles de organizaciones escolares positivas o virtuosas considerando, por un lado, el género y tipo de financiamiento y, por otro, los niveles de bienestar psicológico presentes en adolescentes ecuatorianos. Cabe señalar que el proceso desarrollado toma en cuenta los contextos sociales y culturales de la realidad ecuatoriana.

El presente artículo aborda, en primer lugar, los enfoques y fundamentos teóricos desde los que se realizó la investigación. En esta lógica se presenta a la Psicología Positiva como el marco conceptual del trabajo realizado y el paraguas desde el que se explican los alcances de las variables analizadas, se definen los entendimientos de la virtuosidad percibida en organizaciones escolares y del bienestar psicológico presente en adolescentes. Seguidamente se presenta el diseño metodológico de la investigación, los instrumentos utilizados y los procedimientos empleados. A continuación, se ponen en consideración los resultados alcanzados. Finalmente, se estructuran conclusiones derivadas del estudio y se identifican temas de futuras investigaciones y se establecen algunas limitaciones principales encontradas en la investigación.

\section{Psicología Positiva}

Lupano-Perugini y Castro-Solano (2010) explican el origen y alcance de la Psicología Positiva de la siguiente manera:

El inicio de la Psicología Positiva se señala en 1998 con el discurso inaugural de Martín Seligman como presidente de la Asociación de Psicólogos Americanos (American Psychological Association por sus siglas en inglés) ... Surge como una respuesta a los enfoques de la Psicología centrados en la enfermedad y en la evidencia de las debilidades de los sujetos. La preocupación y el interés por el estudio del bienestar humano y los factores que contribuyen al mismo, no es exclusividad de la Psicología Positiva... Sin embargo, el mérito que corresponde a la Psicología Positiva es haber integrado en un corpus

Licencia Creative Commons Atribución 4.0 Internacional (CC BY 4.0)

Revista Cátedra, 2 (2), pp. 76-93, mayo-agosto 2019. e-ISSN: 2631-2875

https://doi.org/10.29166/catedra.v2i2.1594 
teórico pero con amplia validación empírica los mencionados tópicos de interés (pp. 43-44).

Seligman (2003) plantea la necesidad de avanzar hacia una psicología más positiva cuando señala que "la Psicología no es solo el estudio de la debilidad y el daño, es también el estudio de la fortaleza y la virtud; el tratamiento no es solo arreglar lo que está roto es también alimentar lo mejor de nosotros" (p. 126). Desde la perspectiva expuesta, la Psicología Positiva constituye "el estudio científico de las experiencias positivas, los rasgos individuales positivos, las instituciones que facilitan su desarrollo y los programas que ayudan a mejorar la calidad de vida de los individuos, mientras previene o reduce la incidencia de la psicopatología" (Seligman y Csikszentmihalyi, 2000, p. 5). Por su parte Jiménez (2014) puntualiza que la Psicología Positiva "aporta una visión más completa del ser humano contemplando tanto las emociones negativas como las positivas con el fin de vivir una vida más plena, más comprometida sin perder de vista a la persona como individuo único e irrepetible" (p. 622).

Salanova y Llorens (2016) explican que lo positivo debe ser entendido desde dos dimensiones: "una que se relaciona con la presencia de algo que por su cualidad es positivo, como la alegría, las relaciones positivas, etc.; y otra que tiene que ver con la preferencia, con algo valioso y con sentido" (p. 161). En esta línea, lo positivo se vincula a niveles altos de felicidad en los individuos y en las sociedades desde un punto de vista que rebasa el placer hedónico (inmediato) y se centra en la satisfacción vital con significado (eudonómica). Así mismo es importantes señalar que lo positivo no se visualiza solo como lo opuesto a lo negativo, si no como un mecanismo para conocer e interactuar sobre el desarrollo integral de los seres humanos y sobre su bienestar. Finalmente, lo positivo se relaciona con mejores niveles de rendimiento de los individuos y de las organizaciones en que se desempeñan.

De acuerdo con lo expuesto la Psicología Positiva constituye una disciplina científica que aborda al ser humano considerando sus habilidades, capacidades y emociones positivas que dan lugar a una vida plena y al bienestar integral. Lo positivo, reflejado en el bienestar y la calidad de vida, constituye el objeto de estudio de la Psicología Positiva. Para que el abordaje de lo positivo sea posible esta disciplina se fundamenta en la rigurosidad del método científico aplicado al análisis y comprensión "del funcionamiento psíquico óptimo de individuos, grupos e instituciones" (Castro-Solano, 2012, p. 8). Seligman et al. (2005) identifican tres pilares que configuran la psicología positiva explicándola como "un término paraguas para el estudio de las emociones positivas (vida placentera); los rasgos de carácter positivo (vida comprometida) y el funcionamiento de las organizaciones positivas (vida con significado)" (p. 410); a esos tres pilares se añadió en lo posterior el pilar de los vínculos sociales (relaciones positivas).

El estudio de la escuela, como organización escolar que promueve o limita el desarrollo integral de niñas, niños y adolescentes, se enmarca en el pilar de las organizaciones positivas e involucra al conjunto de actores de la comunidad educativa (tomadores de decisiones, docentes, estudiantes, padres y madres de familia y funcionarios administrativos). La escuela aparece como un espacio privilegiado para generar factores protectores y prevenir factores de riesgo en la adolescencia que posibiliten el desarrollo integral y positivo de chicos y chicas. El diseño e implementación de estrategias e intervenciones socio educativa que consideren variables como la motivación, el liderazgo, la inteligencia emocional, las capacidades metacognitivas, la práctica social, entre otras, contribuirán a alcanzar el objetivo descrito. En palabras de Salanova y Llorens (2016) se entienden como intervenciones positivas (o en Psicología Positiva)" aquellas estrategias 
que son implementadas para mejorar el desarrollo y la satisfacción de las personas con el objetivo final de promover la salud, la calidad de vida y la excelencia" (p. 163).

Castro-Solano (2010) afirma que el rol clásico del psicólogo educativo, desde la psicología tradicional, se ha limitado a la "administración de baterías psicoeducacionales a niños y adolescentes con trastornos de aprendizaje, a la orientación psicológica a padres de jóvenes con problemas y a las tareas de asesoramiento y orientación educativa" (p. 35). A lo señalado habría que añadir el diseño e implementación de programas de escuela para familias y de acciones destinadas a prevenir la violencia entre pares y el consumo de alcohol y drogas; y, en menor escala al apoyo a docentes de aula en el diseño de adaptaciones curriculares de acuerdo con las necesidades de aprendizaje de los estudiantes. Desde una lógica de intervención positiva en la escuela, el Modelo de Funcionamiento de los Departamentos de Consejería Estudiantil tiene 5 ejes clave en el trabajo del psicólogo educativo y del psicopedagogo que tienen que ver con la promoción y prevención del bienestar psicológico, detección de situaciones de riesgo, intervenciones psicopedagógicas, remisión de casos y seguimiento de casos (Ministerio de Educación, 2016, pp.21-34).

\section{Organizaciones escolares positivas (virtuosas)}

El tercer pilar de la Psicología Positiva, de las organizaciones positivas, es abordado desde la Psicología Organizacional Positiva entendida como "el estudio y la aplicación de las capacidades positivas y las fortalezas que pueden ser medidas, desarrolladas y gestionadas eficazmente para mejorar el rendimiento" (Nelson y Cooper, 2007, p. 137). Lupano-Perugini (2017) justifica el aparecimiento de la Psicología Organizacional Positiva al explicar que tradicionalmente la psicología organizacional enfatizó en el abordaje de las situaciones negativas y sus consecuencias "por lo que se requiere de una perspectiva alternativa que garantice resultados óptimos y no se centre únicamente en los aspectos que lo impiden" (p. 37). Por su parte, Salanova, Llorens y Martínez (2016), desde la perspectiva salugénica, definen a la Psicología Organizacional Positiva como:

El estudio científico del funcionamiento óptimo de la salud de las personas y de los grupos en las organizaciones, así como de la gestión efectiva del bienestar psicosocial en el trabajo y del desarrollo de organizaciones para que sean más saludables (p. 177).

Entre los campos de interés de esta tercera vía pueden mencionarse a los principales espacios de socialización de niñas, niños y adolescentes como son la familia, la escuela, el grupo de amigos y la comunidad vistos como instituciones que construyen interacciones positivas. Lupano-Perugini y Castro-Solano (2010) explican que "esta tercera vía es la aplicación de las fortalezas personales para el desarrollo de algo más importante y amplio que uno mismo, es poder aplicar las fortalezas para ayudar a los demás y hacer que estos puedan desarrollar sus potencialidades" (p. 48).

Peterson (2006) identifica a las virtudes en las organizaciones positivas como "las características morales de la organización en su conjunto que van más allá de las características individuales de cada uno de sus miembros; como tales, las virtudes a nivel organizacional son una parte duradera de la cultura organizacional" (p. 1152). LupanoPerugini (2017) enfatiza que "las virtudes organizacionales son definidas como atributos o prácticas morales (e.g. justicia, respeto, dignidad) a nivel global dentro de una organización y no como la suma de las virtudes individuales de sus miembros" (p. 37).

Licencia Creative Commons Atribución 4.0 Internacional (CC BY 4.0)

Revista Cátedra, 2 (2), pp. 76-93, mayo-agosto 2019. e-ISSN: 2631-2875

https://doi.org/10.29166/catedra.v2i2.1594 
La reflexión teórica y la investigación empírica en Psicología Organizacional Positiva determina el uso del término virtudes para definir características individuales vinculadas con fortalezas y valores positivos; mientras que el término virtuosidad hace referencia a características colectivas presentes en las organizaciones definidas como positivas y que son algo más que la suma de las virtudes de sus integrantes. Desde la entrada señalada, Cameron (2012) plantea que:

El término 'virtudes' hace referencia a atributos individuales que representan bondad y excelencia moral, rasgos que indican las mejores cualidades de la humanidad; mientras que el término 'virtuosidad' se refiere al conjunto de virtudes que se manifiestan de manera recurrente como comportamientos, procesos y rutinas en los entornos organizacionales (p. 2).

De acuerdo con lo señalado, el estudio y la promoción de la virtuosidad en las organizaciones definidas como positivas deviene en un propósito principal de la Psicología Organizacional Positiva. La investigación empírica determina que la virtuosidad presenta dos cualidades básicas: amplificación y amortiguación. Lupano-Perugini y Castro-Solano (2018) explican que:

La cualidad amplificadora se relaciona con el hecho de que la exposición a prácticas positivas incrementa los niveles de emociones positivas, capital social y conductas prosociales, lo cual retroalimenta a las prácticas y favorece a la consecuente obtención de mejores resultados organizacionales. Por otro lado, la cualidad amortiguadora se relaciona con ayudar a prevenir efectos negativos, producto de situaciones estresantes, promoviendo en los integrantes un sentido de resiliencia, solidaridad y eficacia (p. 2).

Palomera (2017) puntualiza que las escuelas positivas o con niveles elevados de virtuosidad son aquellas que promueven el bienestar integral y el aprendizaje a partir de acciones como las siguientes: "respeto y valor de la felicidad; metodologías estimulantes, flexibles y variadas; espacios y tiempos para la felicidad; respeto a las voces de niños y adolescentes; actitud de docentes; y, formación del profesorado" (p. 68-69). La construcción de virtudes percibidas en organizaciones escolares consideró cuatro virtudes (gratitud, inspiración, perdón, coraje) de las seis virtudes identificadas para organizaciones laborales por Cameron et al. (2011):

1) Cuidado, entendido como preocupación, interés y responsabilidad por sí mismo y los otros; 2) coraje, referido al apoyo mutuo y valoración por quienes luchan; 3) perdón, entendido como evitar culpabilizar y perdonan errores; 4) inspiración, relacionada con lo que se aprende de los otros); 5) significado vinculado a valoración; y, 6) gratitud visto como integridad, respeto y aprecio (p. 37).

\section{Bienestar psicológico en adolescentes}

La noción de bienestar se encuentra íntimamente ligada a la de salud integral, esta última se entiende como "un estado de completo bienestar físico, mental y social, y no solamente como la ausencia de afecciones o enfermedades" (Organización Mundial de la Salud, 2006, pág. 1). Desde esa perspectiva, el bienestar psicológico constituye uno de los temas de 
interés principal de la Psicología Positiva. Casullo (2002) define al bienestar como "la percepción que una persona tiene sobre los logros alcanzados en su vida, del grado de satisfacción personal con lo que hizo, hace o puede hacer, desde una mirada estrictamente personal" (p. 11).

Romero-Carrasco et al. (2007) explican que dos perspectivas básicas animan la discusión sobre bienestar. La primera de ellas, denominada hedónica, plantea que el bienestar consiste en la "felicidad subjetiva construida sobre la experiencia del placer frente al displacer, incluyendo los juicios sobre los buenos y malos elementos de la vida" (p. 336). La segunda perspectiva, llamada eudoemónica, establece que el bienestar tiene relación con "experiencias relacionadas con sentirse vivo y realizado, con ver la actividad que se realiza como algo que llena y con la impresión de que lo que hacemos tiene sentido" (p. 336). La perspectiva hedónica se vincula con el bienestar subjetivo definido como "una amplia categoría de fenómenos que incluye las respuestas emocionales de las personas, la satisfacción con los dominios, y los juicios globales sobre la satisfacción con la vida" (Diener et al., 1999, p. 277). Por su parte la perspectiva, eudoemónica se relaciona con el bienestar psicológico entendido como "una dimensión fundamentalmente evaluativa que tiene que ver con la valoración del resultado logrado con una determinada forma de haber vivido" (Castro-Solano, 2009, p. 47).

Castro-Solano (2011) hipotetiza "que las rutas relacionadas con el bienestar eudaemónico (vida comprometida y vida con significado) tendrán más relación con la satisfacción vital que la ruta referida al bienestar hedónico (vida placentera)" (pág. 42). Ryff (1989) desarrolla un modelo destinado a explicar el bienestar psicológico relacionado con el funcionamiento positivo de los individuos, dicho modelo se integra por seis dimensiones interrelacionadas entre sí: "1) autoaceptación; 2) relaciones positivas con otros; 3) autonomía; 4) dominio del entorno; 5) propósito en la vida; y, 6) crecimiento personal" (p. 1071).

El bienestar deviene en un constructo triárquico conformado por emociones positivas y emociones negativas lábiles y momentáneas (estados emocionales) y, por un componente cognitivo más estable (bienestar psicológico) que permite a las personas evaluar sobre cómo les va en la vida. Casullo y Castro-Solano (2000) explican que "una persona tiene alto bienestar si experimenta satisfacción con su vida, si frecuentemente su estado anímico es bueno y sólo ocasionalmente experimenta emociones poco placenteras como tristeza o rabia" (p. 37). Desde esta mirada

Tomando como base las propuestas teóricas de Ryff y las conclusiones arrojadas en su propia investigación empírica, Castro-Solano y Casullo (2001) estudian, validan y explican las siguientes dimensiones que configuran el bienestar psicológico en adolescentes: "control de situaciones (sensación de control y autocompetencia), vínculos psicosociales (calidad de las relaciones personales), proyectos (metas y propósitos en la vida) y aceptación de si (sentimiento de bienestar con uno mismo)"(p. 49). Páramo et al. (2012) explican el significado de los puntajes altos en las dimensiones del bienestar psicológicoadolescente:

1) Autonomía: reflejan capacidad para tomar decisiones y evaluar las consecuencias lógicas de las acciones emprendidas; 2) vínculos afectivos (psicosociales): indican una buena capacidad para establecer relaciones sociales basadas en el afecto y en la empatía; 3) propósito con la vida (proyectos): señalan metas y objetivos a ser alcanzados; 4) autoaceptación: se relaciona con las cualidades de autodeterminación, independencia y regulación de la conducta (p. 11).

Licencia Creative Commons Atribución 4.0 Internacional (CC BY 4.0) 


\section{Métodos y materiales}

El estudio que se describe fue de tipo cuantitativo con corte transversal correlacional. Se trabajó con una muestra no probabilística de 550 adolescentes matriculados en el nivel de bachillerato, se persiguió mantener un equilibrio en cuanto al sexo de los participantes por lo que se consideró un $51,3 \%$ de chicos y un $49,7 \%$ de chicas con un promedio de edad de 16 años, 0 meses y una desviación estándar de 4,67. La muestra establecida correspondió a estudiantes de planteles financiados con recursos del Estado ecuatoriano $(71,3 \%)$ y con recursos de los municipios o gobiernos autónomos descentralizados $(28,7 \%)$. Es importante señalar que para el desarrollo de la investigación los estudiantes participaron de forma voluntaria y sus familias dieron su consentimiento informado por medio de un documento firmado.

El instrumento empleado para la medición de virtudes positivas en establecimientos educativos fue la Escala de Virtuosidad Percibida en Organizaciones Escolares construido y validado por Unda-Villafuerte (2018, pp. 95-104) con base en la teoría de organizaciones positivas (virtuosas) y las dimensiones desarrolladas desde esa perspectiva por Cameron et al. $(2011$, p. 6). El instrumento considera una escala tipo Likert de cinco opciones (desde totalmente de acuerdo, de acuerdo, medianamente de acuerdo, poco de acuerdo hasta totalmente en desacuerdo), cuatro factores (gratitud, inspiración, perdón y coraje) y 24 ítems. El instrumento, que responde a los contextos y realidades socio cultural del Ecuador, presenta buenas propiedades de validez y confiabilidad y fue aplicado de manera grupal.

Para la medición del bienestar psicológico en adolescentes se tomó en cuenta la Escala de Bienestar Psicológico en Adolescentes - Ecuador, adaptada por Unda-Villafuerte (2018, pp. 104-111) a partir de un instrumento similar trabajado por Castro-Solano y Casullo (2001, p. 68) en Argentina y la teoría sobre bienestar psicológico en adolescentes desarrollada por Ryff (1989, pp. 1069-1081). El instrumento considera una escala tipo Likert de tres opciones (en desacuerdo, ni de acuerdo ni en desacuerdo, de acuerdo) conformado por 13 ítems y cuatro dimensiones (aceptación, proyectos, vínculos y autonomía). El instrumento, que responde a los contextos y realidad socio cultural del Ecuador, presenta buenas propiedades de validez y confiabilidad y fue aplicado de manera grupal.

A partir de la tabulación de la información levantada se calculó el índice de correlación presente entre las dimensiones correspondientes a virtudes percibidas y las de bienestar psicológico en adolescentes. Seguidamente, mediante el análisis de clúster o conglomerados de tipo $\mathrm{k}$ medias se establecieron grupos vinculados con el bienestar psicológico en adolescentes y, mediante un análisis ANOVA de un factor, se confirmó la distribución de tres grupos seleccionada. A continuación, siguiendo el procesamiento de conglomerados con $\mathrm{k}$ medias y el análisis ANOVA con un factor, se definieron perfiles de organizaciones virtuosas en función de los tipos de bienestar psicológico definidos. El procesamiento de datos y el análisis de la información se llevó a cabo con la ayuda del programa estadístico SPSS 23.0.

\section{Resultados}

El primer proceso estadístico desarrollado tuvo que ver con el análisis correlacional entre las variables virtuosidad percibida en organizaciones escolares y bienestar psicológico en adolescentes ecuatorianos (Cuadro 1), el proceso tomó en cuenta los parámetros establecidos por Cohen (1998) quien plantea como "correlaciones moderadas a los valores iguales o superiores a .30, y como correlaciones pequeñas aquellas mayores a $.25 \mathrm{y}$ menores a .30" (p. 115). Desde la lógica mencionada se observaron correlaciones positivas en todos los cruces con un tamaño del efecto que va de pequeño, en pocos casos, a moderado, en la 
mayoría de los casos. Al analizar las relaciones entre dimensiones se puede observar correlaciones moderadas en el 75 \% de cruces de las dimensiones estudiadas.

\begin{tabular}{|c|c|c|c|c|c|}
\hline \multirow{2}{*}{$\begin{array}{l}\text { Dimensiones } \\
\text { Virtuosidad } \\
\text { (VP) }\end{array}$} & \multicolumn{4}{|c|}{ Dimensiones Bienestar Psicológico (BIEPS) } & \multirow{2}{*}{$\begin{array}{l}\text { BIEPS } \\
\text { (total) }\end{array}$} \\
\hline & Aceptación & Proyectos & Vínculos & Autonomía & \\
\hline Gratitud & $.302^{* *}$ & $.301^{* *}$ & $.451^{* *}$ & $.406^{* *}$ & $.485^{* *}$ \\
\hline Inspiración & $.300^{* *}$ & $.266^{* *}$ & $.393^{* *}$ & $.403^{* *}$ & $.455^{* *}$ \\
\hline Perdón & $.267^{* *}$ & $.303^{* *}$ & $.388^{* *}$ & $.346^{* *}$ & $.434^{* *}$ \\
\hline Coraje & $.243^{* *}$ & $.275^{* *}$ & $.360^{* *}$ & $.301^{* *}$ & $.394^{* *}$ \\
\hline VP (Total) & $.346^{* *}$ & $.353^{* *}$ & $.498^{* *}$ & $.457^{* *}$ & $.551^{* *}$ \\
\hline
\end{tabular}

$* * \mathrm{p}<.01$ bilateral.

Cuadro1: Correlación entre las variables virtuosidad percibida y bienestar psicológico en adolescentes

Una vez comprobada la correlación entre las variables de estudio se identificaron clúster (tipo k media) que den cuenta de una potencial distribución de los niveles bienestar psicológico (cuadro 2); dichos conglomerados consideraron como variables transversales al género de los participantes (masculino, femenino) y al tipo de organización escolar (municipal, fiscal). Es así como se definieron los siguientes grupos o conglomerados: (i) bienestar psicológico fortalecido, corresponde al $58 \%$ de casos de la muestra con una mayor presencia de mujeres que de hombres; (ii) bienestar psicológico en construcción, corresponde al $36 \%$ de casos de la muestra con una mayor presencia de hombres que de mujeres; y, (iii) bienestar psicológico vulnerable; corresponde al $6 \%$ de casos de la muestra con una mayor presencia de hombres que de mujeres. En los tres grupos identificados predominó la presencia de chicas y chicos que provienen de establecimientos fiscales.

\begin{tabular}{lrrrrr}
\hline & & \multicolumn{2}{c}{ Bienestar Psicológico } & & Total \\
& & $\begin{array}{c}\text { Fortalecido } \\
(n=320)\end{array}$ & $\begin{array}{c}\text { En } \\
\text { construcción } \\
(n=200)\end{array}$ & $\begin{array}{c}\text { Vulnerable } \\
(n=30)\end{array}$ & \\
\hline Género & Masculino & $42.5 \%$ & $63.3 \%$ & $62.0 \%$ & 279 \\
& Femenino & $57.5 \%$ & $36.7 \%$ & $38.0 \%$ & 271 \\
Organización & Fiscal & $78.2 \%$ & $70.0 \%$ & $69.0 \%$ & 392 \\
educativa & Municipal & $27.2 \%$ & $30.0 \%$ & $31.0 \%$ & 158 \\
\hline
\end{tabular}

Cuadro 2: Conglomerados de bienestar psicológico en adolescentes distribuidos por género y tipo de organización educativa (porcentajes)

Se verificaron modelos con tres, cuatro y cinco grupos en los que se clasificó el nivel de bienestar psicológico; finalmente, se optó por un modelo conformado por tres grupos por ser el que mejores distribuciones de las medidas descriptivas presentaba (media aritmética y desviación estándar). Lo mencionado se confirmó con el análisis ANOVA (cuadro 3), mismo que incluyó como variables dependientes a las dimensiones del bienestar psicológico (aceptación, proyectos, vínculos y autonomía) y como factor de pertenencia a 
los conglomerados vinculados al nivel de bienestar psicológico en adolescentes, definidos en un primer momento (bienestar psicológico fortalecido, en construcción y vulnerable).

\begin{tabular}{lcccc}
\hline $\begin{array}{c}\text { Dimensiones } \\
\text { Bienestar } \\
\text { Psicológico }\end{array}$ & $\begin{array}{c}\text { Fortalecido } \\
(n=320) \\
\text { Media }(D S)\end{array}$ & $\begin{array}{c}\text { En construcción } \\
(n=200)\end{array}$ & $\begin{array}{c}\text { Vulnerable } \\
(n=30)\end{array}$ & ANOVA \\
& $11.36(.75)$ & $10.06(1.15)$ & $7.17(1.42)$ & $326.349^{* *}$ \\
\hline Aceptación & $8.42(.70)$ & $7.63(1.05)$ & $6.17(1.56)$ & $113.701^{* *}$ \\
Proyectos & $8.41(.64)$ & $7.22(1.15)$ & $5.90(1.45)$ & $176.516^{* *}$ \\
Vínculos & $8.03(.79)$ & $6.80(.91)$ & $5.00(1.02)$ & $258.529^{* *}$ \\
Autonomía & & & & \\
\hline
\end{tabular}

$* * \mathrm{p}<.001$

Cuadro 3: Análisis unidireccional de varianzas (ANOVA) vinculados los conglomerados que caracterizan el bienestar psicológico de adolescentes

Con los insumos obtenidos se avanzó en la definición de perfiles que vinculen a las organizaciones escolares positivas con los conglomerados referidos al nivel de bienestar psicológico en adolescentes definidos previamente (cuadro 4). En esta perspectiva se desarrolló un análisis ANOVA unifactorial en donde las variables dependientes fueron los puntajes de las dimensiones de la virtuosidad percibida en organizaciones escolares y el factor de pertinencia los conglomerados en que se agrupó el bienestar psicológico en adolescentes. En todos los cruces se evidenciaron diferencias significativas en el valor de las medias.

\begin{tabular}{lcccc}
\hline $\begin{array}{l}\text { Dimensiones } \\
\text { Virtuosidad } \\
\text { Percibida }\end{array}$ & $\begin{array}{c}\text { Fortalecido } \\
(n=320) \\
\text { Media (DE) }\end{array}$ & $\begin{array}{c}\text { Bienestar Psicológico } \\
\text { En construcción } \\
(n=200)\end{array}$ & $\begin{array}{c}\text { Mulnerable } \\
(n=30)\end{array}$ & ANOVA \\
\hline Gratitud & $35.72(4.50)$ & $31.89(6.15)$ & $26.73(8.71)$ & $57.49^{* *}$ \\
Inspiración & $22.98(3.36)$ & $20.86(3.61)$ & $17.40(5.46)$ & $46.78^{* *}$ \\
Perdón & $19.53(3.30)$ & $17.26(3.39)$ & $17.70(4.78)$ & $46.46^{* *}$ \\
Coraje & $14.63(2.68)$ & $13.30(2.67)$ & $11.33(3.83)$ & $29.08^{* *}$ \\
\hline
\end{tabular}

${ }^{* *} \mathrm{p}<.001$

Cuadro 4: Conglomerados de bienestar psicológico y su relación con la virtuosidad percibida en organizaciones escolares

Con el apoyo de la información empírica levantada se establecieron tres perfiles que caracterizaron a las organizaciones escolares estudiadas, estos se definen de la siguiente manera: 
- Establecimientos educativos percibidos como virtuosos con estudiantes que demuestran niveles de bienestar psicológico fortalecidos: en este perfil se ubicó el $58 \%$ de casos de la muestra (57,5\% mujeres). Se trató de organizaciones escolares positivas que inspiraban con el ejemplo de sus integrantes el esfuerzo y la dedicación, promovían la gratitud, el reconocimiento y el perdón cuando ameritaba y establecían el alcance de logros a partir del esfuerzo, la decisión y el coraje. Sus miembros demostraban contar con planes de vida, evidenciaban autonomía en la toma de decisiones, las relaciones entre pares y con adultos se sustentaba en la empatía y en la comunicación asertiva y en la aceptación de las propias fortalezas y debilidades.

- Establecimientos educativos percibidos como en tránsito a la virtuosidad con estudiantes que presentaban niveles de bienestar psicológico en construcción: este grupo aglutinó al $36 \%$ de casos presentes en la muestra (36,7 \% mujeres). Este perfil se caracterizó por adolescentes que avanzaban con tropiezos en la construcción de sus fortalezas y valores permitiendo su adaptación a situaciones nuevas y cambiantes en ambientes educativos cuyas normas y políticas aún eran difusas y requerían ser definidas con mayor claridad. La gratitud, el perdón y el sentido de trascendencia se evidenciaba de manera ocasional en la organización escolar y los adolescentes requerían apoyo para fijar metas y objetivos y plasmarlos en planes o proyectos de vida, así como para tomar decisiones y dirigir su accionar a logros positivos.

- Establecimientos educativos percibidos como poco virtuosos con estudiantes que presentan niveles de bienestar psicológico vulnerable: convergieron en este perfil el $6 \%$ de casos de la muestra (38\% mujeres). Los adolescentes ubicados en este perfil presentaban dificultades para establecer metas y objetivos a corto y mediano plazo, la interacción social productiva y las actitudes de cuidado y autocuidado eran débiles presentando conductas de riesgo. Para este grupo el perdón, la gratitud o la espiritualidad eran valores no incorporados, no se definían con claridad proyectos de vida y tampoco consideraban que la organización escolar inspiraba sus prácticas y valores de apoyo, solidaridad y compasión.

\section{Conclusiones}

Si bien el estudio descrito evidencia una relación entre virtuosidad percibida en organizaciones escolares y bienestar psicológico en adolescentes que se inscribe en un nivel moderado, es posible afirmar que organizaciones que practican la gratitud, el perdón, el coraje y son fuente de inspiración inciden de manera directa en mejores niveles de autonomía en adolescentes, en su capacidad para planificar el futuro, en la aceptación de sí mimos y de los demás y en el establecimiento de vínculos positivos y duraderos. De esta forma, todas las dimensiones de la variable organizaciones virtuosas percibidas aportan en el esfuerzo deliberado por alcanzar mejores niveles de bienestar psicológico (eudaemónico). Lo mencionado confirma la validez teórica de la investigación realizada y la interacción entre las variables de estudio.

La investigación desarrollada determina que las adolescentes mujeres presentan mejores niveles de bienestar psicológico que los varones; esto se relaciona además con niveles más altos de virtuosidad percibida en las organizaciones escolares a las que asisten. Lo mencionado refiere un interés más sostenido por parte de las chicas en comparación con los chicos para proyectarse hacia el futuro y proyectar sus vidas a mediano y largo plazo, aceptarse a sí mismas como individuos integrales, buscar y mantener relaciones positivas y duraderas y demostrar mayor capacidad para tomar decisiones con autonomía. Lo mencionado se potencia en espacios escolares virtuosos donde encuentran modelos 
inspiradores a seguir, en ambientes que promueven la gratitud como valor principal, en contextos en los que se reconocen los errores y en ambientes retadores que impulsan la consecución de logros.

Es necesario recalcar que las adolescentes mujeres valoran en alto grado organizaciones escolares donde "la práctica de valores como el respeto y la solidaridad son hechos cotidianos, en las que la inclusión y las oportunidades de desarrollo son permanentes, que brindan apoyo psicoemocional y que cuentan con docentes capacitados" (Unda-Villafuerte, 2018, p. 119). En la lógica mencionada una línea de investigación futura deberá relacionar cómo las organizaciones escolares definidas como virtuosas desarrollan capacidades en adolescentes mujeres y varones para hacer frente de manera resiliente y eficaz a los riesgos psicosociales a los que se hallan expuestos tales como la violencia por razones de género, acoso escolar, presión para el consumo de alcohol y otras drogas, suicidio, situaciones de movilidad humana, entre otros.

La identificación de perfiles de organizaciones escolares virtuosas en relación con los niveles de bienestar psicológico en adolescentes constituye un aporte importante de este estudio. Trabajar desde la gratitud, el perdón, el coraje o la inspiración como dimensiones de la virtuosidad escolar; así como en la autonomía, vínculos, proyectos y aceptación, como dimensiones del bienestar psicológico, aseguran a los adolescentes contar con factores protectores para hacer frente a los riesgos psicosociales. De esta forma se plantean tres niveles de organizaciones escolares (establecimientos educativos virtuosos, en tránsito a la virtuosidad y poco virtuosos) que interactúan en su orden con tres niveles de bienestar psicológico en adolescentes (bienestar psicológico fortalecido, en proceso de construcción y vulnerable). Los tipos de organizaciones escolares y los niveles de bienestar psicológico en adolescentes deberán ser revisados, ajustados, replanteados y aplicados en el trabajo con docentes, autoridades, familias y con los propios adolescentes.

La virtuosidad percibida deviene en un marcador de calidad educativa en organizaciones escolares determinada por la capacidad de responder de manera eficiente y oportuna a las necesidades de desarrollo cognitivo, afectivo y social de chicos y chicas y, por tanto, a su valoración personal y social reflejada en las dimensiones e indicadores que configuran el bienestar psicológico. Para que lo mencionado sea posible, las dimensiones de la virtuosidad percibida en organizaciones escolares deben concretarse e institucionalizarse a través de los estándares de gestión escolar y sus dimensiones definidos por el Ministerio de Educación del Ecuador (2017, p.9):

1. Gestión administrativa: organización institucional, desarrollo profesional, información y comunicación, infraestructura, equipamiento y servicios complementarios.

2. Gestión pedagógica: componente enseñanza aprendizaje, consejería estudiantil y refuerzo pedagógico.

3. Convivencia, participación escolar y cooperación: convivencia y participación escolar y alianzas estratégicas de cooperación para el desarrollo.

4. Seguridad escolar: gestión de riesgos y protección.

Es decir, la construcción de organizaciones escolares positivas percibidas como virtuosas y el desarrollo de niveles aceptables de bienestar psicológico en adolescentes deberán estar ligados a los estándares de calidad establecidos para la educación del Ecuador. En las organizaciones escolares, los estándares e indicadores de calidad educativa equivalen a los indicadores de performance o rendimiento de las organizaciones de trabajadores. Si un 
supuesto clave es que las organizaciones virtuosas mejoran el rendimiento institucional, una investigación posterior debería abordar cómo las organizaciones escolares percibidas como virtuosas inciden en mejorar los estándares de calidad educativa previamente establecidos por la autoridad educativa nacional.

En síntesis, la investigación desarrollada da cuenta de que más altos niveles de felicidad y satisfacción vital en adolescentes están ligados a entornos escolares virtuosos que estimulan el desarrollo de fortalezas y valores personales y sociales y de normas, políticas, planes y programas educativos que potencian el bienestar psicológico. A la vez, mejores niveles de bienestar psicológico en chicos y chicas permiten construir organizaciones escolares virtuosas. Sin embargo, lo mencionado no es suficiente, la investigación social determina que la felicidad y el bienestar se encuentran ligados a mejores condiciones de vida reflejadas en el ejercicio de derechos, en la igualdad entre los géneros, la inclusión desde la diversidad y la satisfacción integral de las necesidades básicas. De esta forma, se trata de generar una nueva escuela y un pacto social en torno a la educación en el marco de un proyecto global de construcción de relaciones sociales y económicas justas y equitativas. Lo mencionado involucra vincular a la escuela con la comunidad y las instituciones que en ella actúan desde el accionar intersectorial e interinstitucional.

Debe indicarse que un limitante del estudio constituye el uso de instrumentos con escalas de autoreporte que pueden ser manipulados en su llenado. Otro limitante tiene que ver con que se trató de una muestra seleccionada de manera no probabilística lo que puede generar sesgos de representatividad. Adicionalmente, debe destacarse que la muestra considera planteles fiscales y municipales, es necesario verificar los resultados y conclusiones en organizaciones escolares con financiamiento privado.

Finalmente, el estudio desarrollado posiciona a la Psicología Positiva como un marco teórico referencial alternativo y disciplinar científico para el entendimiento de la escuela como organización positiva y del accionar de los actores de la comunidad educativa (estudiantes, familias, tomadores de decisiones) desde su valores, fortalezas y bienestar. Se plantea además el reto de generar programas, que construidos desde la Psicología Positiva, posibiliten el desarrollo integral de los actores de los procesos de educación y de sus instituciones. 


\section{Bibliografía}

Barcelata, B. y Rivas, G. (2016). Bienestar psicológico y satisfacción vital en adolescentes mexicanos tempranos y medios. Revista Costarricense de Psicología. Julio Diciembre, volumen 35, número 2, 119 - 137. ISNN 0257-1439.

Cameron, K. S. (2012). Virtuousness and performance: a productive partnership. Execitive white paper series. Michigan Ross Scholl of Business.

Cameron, K., Mora, C., Leutscher, T. y Calarco, M. (2011). Effects of Positive Practices on Organizational Efectiveness. The Journal of Applied Behavioral Science XX(X) 1- 3.

Castro-Solano, A. (2009). El bienestar psicológico: cuatro décadas de progreso. Revista Interuniversitaria de Formación del Profesorado, 23 (3), 43-72.

Castro-Solano, A. (2010). Fundamentos de Psicología Positiva. Paidós: Buenos Aires.

Castro-Solano, A. (2011). Las rutas de acceso al bienestar. Relaciones entre bienestar hedónico y eudaemónico. Un estudio en población argentina. Revista Iberoamericana de Diagnóstico y Evaluación. vol. 1, núm. 31, pp. 37-57.

Castro-Solano, A. (2012). La Psicología Positiva en América Latina. Desarrollos y perspectiva. PSIENCIA, Revista Latinoamericana de Ciencia Psicológica, 4 (2), 108 118.

Castro-Solano, A. y Casullo, M. M. (2001). Evaluación del bienestar psicológico en estudiantes adolescentes argentinos. Revista de Psicología. Lima, Universidad Católica del Perú, XVIII (2), 35 - 68.

Casullo, M. M. (2002). Evaluación del Bienestar Psicológico en Iberoamérica. Buenos Aires: Paidós.

Casullo, M. M., y Castro-Solano, A. (2000). El significado de bienestar en estudiantes adolescentes. Revista Iberoamericana de Diagnóstico y Evaluación - e Avaliação Psicológica, 12(2), 57-70.

Cohen, J. (1988). Statistical power analysis for the behavioral sciences. (2. ${ }^{\mathrm{a}}$ Ed), Erlbaum, Hillsdale, NJ.

Diener, E., Suh, E.M., Lucas, R.E. \& Smith, H.L. (1999). Subjective well-being: Three decades of progress. Psychological Bulletin, 125, 276-302.

Fondo de Naciones Unidas para la Infancia (UNICEF). (2011). La adolescencia una época de oportunidades. Fondo de las Naciones Unidas para la infancia. New York.

González, S. y Rivera, M. (2016). Efectos del apoyo psicosocial, resiliencia y competencia social en el bienestar psicológico de adolescentes de sectores vulnerables, con eventos de vida estresantes. En Gaxiola, J. \& Palomar, J. (coordinadores). El bienestar psicológico, una mirada desde Latinoamérica, 31-60. Universidad de Sonora. Qartuppi. Primera edición. Hermosillo, México.

Güemes-Hidalgo, M., Ceñ al González, M. e Hidalgo-Vicario, M. (2017). Pubertad y adolescencia. Adolescere. Revista de Formación Continuada de la Sociedad Española de Medicina de la Adolescencia. Volumen V. Enero-Febrero 2017. No 1. 
Jiménez, V. (2014). Psicología contemporánea básica y aplicada. Psicología Positiva. Ediciones Pirámide. Capítulo 21, 621-645.

Lupano-Perugini, M. L. (2017). Virtudes organizacionales, performance y satisfacción laboral. Diferencias según variables individuales y organizacionales en empleados argentinos. Psicodebate Vol. 17 No 1 ISSN: 1515-2251 e-ISSN: 2451-6600, 35-50 http://dx.doi.org/10.18682/pd.v17i1.637

Lupano-Perugini, M. L. y Castro-Solano, A. (2010). Psicología Positiva: análisis desde su surgimiento. Cienc. Psicol. [online]. vol.4, n.1, pp.43-56. ISSN 1688-4221.

Lupano-Perugini, M. L. y Castro-Solano, A. (2018). Influencia de las virtudes organizacionales sobre la performance laboral. Un estudio en organizaciones argentinas. Centro Interamericano de Investigaciones Psicológicas y Ciencias Afines (CIIPCA), volumen 35.

Ministerio de Educación del Ecuador (2016). Modelo de Funcionamiento de los Departamentos de Consejería Estudiantil. Quito-Ecuador

Ministerio de Educación del Ecuador (2017). Manual para la implementación y evaluación de los estándares de calidad educativa: gestión escolar, desempeño profesional directivo y desempeño profesional docente. Subsecretaría de Fundamentos Educativos. Dirección Nacional de Estándares Educativos. Quito, Ecuador. ISBN: 978-9942-22-234-3

Navarro-Pérez, J., Pérez-Cosín, J., y Perpiñán, S. (2015). El proceso de socialización de los adolescentes postmodernos: entre la inclusión y el riesgo. Recomendaciones para una ciudadanía sostenible. Pedagogía Social. Revista Interuniversitaria, (25), 143170.

Nelson, D., y Cooper, C. (2007). Positive organizational behavior: Accentuating the positive at work. Thousand oaks, CA: Sage.

Organización Mundial de la Salud. (2006). Constitución de la Organización Mundial de la Salud. Documentos básicos, suplemento de la $45^{\mathrm{a}}$ edición.

Palomera, M. (2017). Psicología Positiva en la Escuela: Un Cambio con Raíces Profundas. Papeles del Psicólogo, 38 (1), 67-71. Recuperado de http://www.papelesdelpsicologo.es/pdf/2823.pdf

Páramo, M., Straniero, C., García, C., Torrecilla, N., y Escalante, E. (2012). Bienestar psicológico, estilos de personalidad y objetivos de vida en estudiantes universitarios. Pensamiento Psicológico, 10 (1).

Peterson, C. (2006). A primer in positive psychology. New York, NY: Oxford University Press. ISBN 9780195188332.

Ryff, C. (1989). Happiness is everything, or is it? Exploration son the meaning of psychological well- being. Journal of Personality and Social Psychology, 57 (6), 10691081.

Romero-Carrasco, A. y Brustad, R. y García Mas, A. (2007). Bienestar psicológico y su uso en la psicología del ejercicio, la actividad física y el deporte. Revista Iberoamericana de Psicología del Ejercicio y el Deporte, 2 (2), 31-52. 
Salanova, M., Llorens, S. (2016). Hacia una psicología positiva aplicada. Papeles del Psicólogo. Psychologist Papers, Vol. 37(3), pp. 161-164.

Salanova, M., Llorens, S. y Martínez, I. (2016). Aportaciones desde la Psicología Organizacional Positiva para desarrollar organizaciones saludables y resilientes. Papeles del Psicólogo, vol. 37 (39), pp. 137-184.

Seligman, M. E. P. (2003). Positive psychology. Fundamental assumptions. American Psychologist, 126-127.

Seligman, M. E. P., Steen, T. A., Park, N., Paterson, C. (2005). Positive psychology progress: Empirical validation of interventions. Fundamental assumptions. American Psychologist, 60 (5), 410-421.

Seligman, M. E. P. y Csikszentmihalyi, M. (2000). Positive psychology: An introduction. American Psychologist, 55, 5-14.

Unda-Villafuerte, F. (2018). Virtuosidad percibida en organizaciones escolares, bienestar psicológico en adolescentes y liderazgo auténtico en docentes. Universidad de Palermo. Tesis doctoral. Repositorio. 


\section{Autores}

FERNANDO UNDA-VILLAFUERTE obtuvo su título de Doctor en Psicología (PhD) por la Universidad de Palermo, Argentina en 2018. Alcanzó el título de Magíster en Desarrollo de la Inteligencia y Educación en 2011, el diplomado en Pedagogías Innovadoras en 2010 y la Especialidad en Diagnóstico Intelectual en 2010 en la Universidad Técnica Particular de Loja. En el año 2001 obtuvo el título de Doctor en Psicología Educativa especialidad en Psicología del Adolescente por la Universidad Central del Ecuador. En 1993 obtuvo el título de Licenciado en Ciencias de la Educación especialidad Psicología Educativa por la Universidad Central del Ecuador.

Actualmente se desempeña como especialista en educación y comunicación para la promoción de la salud en el Ministerio de Salud Pública del Ecuador. Es profesor contratado del Instituto de Investigación y Posgrado de la Facultad de Filosofía, Letras y Ciencias de la Educación de la Universidad Central del Ecuador desde el 2013. Ha sido profesor titular de la Escuela de Psicología de la Universidad de las Américas en 2014. En la organización internacional no gubernamental CARE se desempeñó como director para Ecuador entre 2012 y 2017, como Gerente de Programas entre 2010 y 2012, además realizó funciones de coordinación y asesoría en educación entre 2002 y 2010. En Plan International Ecuador fue gerente de la Unidad de Programas Quito entre 2000 y 2002 y desempeñó funciones de coordinación en educación para la oficina de Plan en la provincia de Bolívar entre 1996 y 2000. Fue coordinador Pedagógico de la Fundación Hoy en la Educación de Diario Hoy entre 1992 y 1996. Entre sus líneas de investigación y estudio destacan las organizaciones escolares positivas, el liderazgo auténtico en docentes y el bienestar psicológico en adolescentes abordados desde la Psicología Positiva, además aborda las temáticas de desarrollo integral del adolescentes y movilidad humana desde los enfoques de inclusión, género e interculturalidad.

MARÍA LAURA LUPANO-PERUGINI es Doctor de la Universidad de Palermo. Phillosophiae Doctor, Ph. D. Tiene el título de Licenciada en Psicología y de Profesora en Nivel Medio y Superior en Psicología por la Universidad de Buenos Aires, Becaria y actualmente investigadora asistente del Consejo Nacional de Investigaciones Científicas y Técnicas (CONICET) de la República de Argentina. Investigadora del Centro de investigaciones de la Facultad de Ciencias Sociales de la Universidad de Palermo.

Entre sus líneas de investigación y estudio destacan el estudio de las organizaciones positivas, el bienestar, los valores y el rendimiento (performance) desde la Psicología Organizacional Positiva. Ha participado en más de 30 reuniones y foros científicos con ponencias referidas a investigación y evaluación en psicología. Sus artículos científicos se encuentran en las más importantes revistas indexadas de la región (Scopus, Latindex, Redalyc, Scielo). 